\title{
USER INTERFACE FOR ANALYSIS OF EXPERIMENTAL DATA
}

\author{
K. Yankov*, D. Ilieva \\ Faculty of Technics and Technologies, Trakia University, Yambol, Bulgaria
}

\begin{abstract}
The nature and the characteristics of the data obtained in scientific experiments is analyzed. The general regularities are formalized and the requirements for the user interface are formulated. Data structures TExperiment and TBasePoint, according to the requirements of Object-oriented programming, are proposed. The approach is implemented with the software Korelia-Ident for identification of experimental data.
\end{abstract}

Key words: data structures, modeling, data processing, object-oriented programming

\section{INTRODUCTION}

The experiment is purposeful activity to obtain data about the nature and behavior of the studied systems. It is implemented in strict conditions which guarantee controlled impact on the system and abilities to receive the result. The impact force is implemented with the parameters that cannot be changed by the system. That is why they are called "independent parameters". The system responds to the force by changing some of its inherent characteristics, which are called "dependent variables". The experimental data can be processed and evaluated with different applied software, which contributes to the freedom and the adaptability of the investigation. The modern devices used in researches can transmit data to computers. The external devices that produce the primary data are known as Data Circuit terminating Equipment (DCE), and the computer and the software that accept and process the data from the devices will be referred to as Data Terminal Equipment (DTE). DCE and DTE are connected by an interface for data transmission: RS 232, USB or LAN. A system for interactive description of the protocols for data transmission from DCE and their acceptance from the user software is described in [1]. In many cases, DCE does not have an interface which can be connected to the DTE. Another reason for the lack of such an

*Correspondence to: Kaloyan Yankov, Trakia University, Faculty of Technics and Technologies 38 Graf Ignatiev str., 8600 Yambol, Bulgaria kaloyan.yankov@trakia-uni.bg interface is the methodology of the study. These are classes of studies that are collected in a long time intervals. In such cases it is appropriate to develop interactive tools for their input.

The purpose of the experiment is to determine and describe the causality between the independent and dependent parameters. It is therefore important to analyze the nature and the characteristics of the independent and dependent variables in different in nature experiments, to establish and formalize the general laws. Formalization will aid in the creation of generalized data structures, and therefore in the creation of dialog procedures for the data input into the programming system.

The purpose of this work is to identify and formalize the general regularities in describing the results of the experiment. This will help create generalized data structures and userfriendly interface of the programming system.

\section{FORMAL DESCRIPTION OF THE EXPERIMENT}

Experimental studies, regardless of the method of conducting and nature of the object of study, have common characteristics. These characteristics may be formalized into procedures which follow a precondition for unification of post-processing and an analysis of the results.

1. The system $S$ is the studied object. The system is treated with an independent variable $\boldsymbol{\Gamma}$ - input influence. 
2. The reaction $\boldsymbol{\Lambda}$ of the system $\boldsymbol{S}$ is:

$\Lambda=S(\Gamma)$

3. The independent variable $\boldsymbol{\Gamma}$ has a set of bounded real values $X_{\Gamma} \subset R$.

4. The dependent variable $\boldsymbol{\Lambda}$ has a co-domain $Y_{\Lambda} \subset R$, belonging to the real numbers.

5. The set $X_{\Gamma}$ possess an extreme additive measure $\boldsymbol{\mu}$, such that:

$$
\begin{aligned}
& \mu_{\Gamma}\left(X_{\Gamma}\right) \geq 0 \\
& \mu_{\Gamma}\left(x_{i}+x_{j}\right)=\mu_{\Gamma}\left(x_{i}\right)+\mu_{\Gamma}\left(x_{j}\right) \\
& \mu_{\Gamma}(\circ)=0 \\
& x_{i} \in X_{\Gamma}, x_{j} \in X_{\Gamma}
\end{aligned}
$$

4. For the set of values $X_{\Gamma}$ of the independent variable is defined a standard $e_{\Gamma}$. Thus for $\forall x \in X_{\Gamma}$ a real number $\boldsymbol{a}$ is assigned :

$$
\mu_{\Gamma}(x)=a . e_{\Gamma}
$$

5. The standard $e_{\Gamma}$ and the measure $\boldsymbol{\mu}$ are applied to obtain $N$ discrete values which will be used to influence the system and observe its behavior. For this purpose $N$ distinct real numbers $\boldsymbol{a}_{1}, \boldsymbol{a}_{2}, \ldots, \boldsymbol{a}_{N}$ are chosen to receive the input's effects:

$$
\mid \begin{aligned}
& x_{1}=a_{1} \cdot e_{\Gamma} ; x_{2}=a_{2} \cdot e_{\Gamma} ; \ldots ; x_{N}=a_{N} \cdot e_{\Gamma} \\
& x_{i} \in X_{\Gamma}, i=1,2, \ldots, N
\end{aligned}
$$

6. With the selected relation $\mathfrak{R}$, the scheme (3) allows to arrange the elements in the set
$X_{\Gamma}$, and they may be numbered according to their position in the order:

$$
\begin{aligned}
& X_{\Gamma}=\left\{x_{1}, x_{2}, x_{3}, \ldots, x_{N-1}, x_{N}\right\} \\
& \mathfrak{R}:[1: N] \rightarrow X_{\Gamma}
\end{aligned}
$$

The most commonly used relation is ' $<$ '.

$$
<: x_{1}<x_{2},<x_{3}<\ldots<x_{N-1}<x_{N}
$$

Thus the domain of $\boldsymbol{X}_{\boldsymbol{\Gamma}}$ is $\left[\mathrm{x}_{1}, \mathrm{x}_{\mathrm{N}}\right]$.

7. There is no requirement for equality of the intervals between the values of the independent parameter, i.e. in general:

$\left(x_{2}-x_{1}\right) \neq\left(x_{3}-x_{2}\right) \neq \ldots \neq\left(x_{N}-x_{N-1}\right)$

8. For each value $x_{i} \in X_{\Gamma}, \boldsymbol{m}_{i}=\left|\boldsymbol{x}_{i}\right|$ a number of effects are applied to the system:

$$
x_{i 1}, x_{i 2}, x_{i 3}, x_{i 4} \ldots, x_{i\left(m_{i}-1\right)}, x_{i m_{i}}
$$

The aim is to explore the statistical parameters of the system's response to a unique value of the input impact. Because of that, almost always assume that the elements of the row (4) are equal. However, this assumption cannot exist in reality. There is a natural dispersion of the independent parameter. This necessitates statistical evaluation of the input values. I.e. the independent parameter is represented by the ordered pair of mean and standard deviation: $\left(\bar{x}_{i}, \sigma_{x i}\right)$. Thus, as a result of conducting an experiment was prepared by a number of ordered pairs representing the independent variable:

$$
\left(\bar{x}_{1}, \sigma_{x, 1}\right),\left(\bar{x}_{2}, \sigma_{x, 2}\right),\left(\bar{x}_{3}, \sigma_{x, 3}\right) \ldots,\left(\bar{x}_{N-1}, \sigma_{x, N-1}\right),\left(\bar{x}_{N}, \sigma_{x, N}\right)
$$

9. For each member of the row (5), $\boldsymbol{m}_{i}$ reactions $y_{i}$ are measured. That is the dependent parameter of the system:

$$
\begin{aligned}
& y_{i, m_{i}}=S\left(x_{i, m_{i}}\right) \\
& i=1,2, \ldots, N, m_{i}=1,2, \ldots,\left|x_{i}\right|
\end{aligned}
$$

That way for the $\boldsymbol{i}$-th input impact $\boldsymbol{x}_{\boldsymbol{i}}$ a row of output responses is received:

$$
y_{i, 1}, y_{i, 2}, \ldots \ldots \ldots, y_{i, m_{i}-1}, y_{i, m_{i}} \in Y_{i} \subset Y_{\Lambda}
$$

$\boldsymbol{Y}_{\boldsymbol{i}}$ - range of the $\boldsymbol{i}$-th dependent parameter.

It is assumed that the values in the row (6) have a Gaussian distribution and can be presented with an average value $\overline{y_{i}}$ and a standard deviation $\sigma_{y i}$. These two variables form the ordered pair $\left(\overline{y_{i}}, \sigma_{y i}\right)$ represent the result of the $\boldsymbol{i}$-th experiment.

The corresponding row of (5) experimental data is:

$\left(\bar{y}_{1}, \sigma_{y 1}\right),\left(\bar{y}_{2}, \sigma_{y 2}\right),\left(\bar{y}_{3}, \sigma_{y 3}\right) \ldots,\left(\bar{y}_{N-1}, \sigma_{y N-1}\right),\left(\bar{y}_{N}, \sigma_{y N}\right)$

Based on the aforesaid, an experiment $\boldsymbol{E}$ can be defined as a relation

$$
\mathrm{E}: \quad \begin{aligned}
& S:\left(\bar{x}_{i}, \sigma_{i x}\right) \rightarrow\left(\bar{y}_{i}, \sigma_{i y}\right) \\
& x_{i} \in X_{\Gamma} \subseteq\left[\bar{x}_{1} \pm \sigma_{x 1}, \bar{x}_{N} \pm \sigma_{x N}\right], \\
& y_{i} \in Y_{\Lambda} \subseteq\left[\inf \left(\bar{y}_{i} \pm \sigma_{y i}\right), \sup \left(\bar{y}_{i} \pm \sigma_{y i}\right)\right] \\
& \mathrm{i}=1,2, \ldots, \mathrm{N}
\end{aligned}
$$

The ordered quaternion:

$\left(\left(\bar{x}_{i}, \sigma_{i x}\right),\left(\bar{y}_{i}, \sigma_{i y}\right)\right)$

will be called experimental base point or base element. 


\section{OBJECT-ORIENTED STRUCTURE OF THE INTERFACE MODEL}

When creating a model of experiment, the possible data categories which the model must reflect have to be considered. These are mainly graphical and non-graphical data. Graphics present experimental data in Euclidean space [2]. Non-graphical data is data of general type, as well as data which characterizes each copy of the experiment. It complements the
YANKOV K., et al. graphical data. Most frequently, the data is either numeric or symbolic. The creation of an object-oriented structure "class of experiment" must take in account these two categories. Data-oriented graphical visualization is described in the abstract class TMicroGraph. TMicroGraph is defined in [3] as an abstract class-ancestor of graphical classes. It has been created as a successor of the base class TObject (Figura 1).

\begin{tabular}{|cl|}
\hline TMicroGraph $=$ class(TObject) & \\
UnicCode : longint; & $\begin{array}{l}\text { // Unic identification code of the } \\
\text { object }\end{array}$ \\
UserIdent : string; & // User defined identificator \\
MicroGraphValid : byte; & // validity flag \\
LinearMetric: & // Pixel, Millimeter, Centimeter, \\
TLinearMetric; & Meter \\
AngleMetric : & // Degree, Radian \\
TAngleMetric; & \\
FVisible : boolean; & // visibility \\
ObjColor : TColor; & // object color \\
.............. & \\
\hline
\end{tabular}

Figure 1. Abstract graphical class-ancestor

Because this class contains the basic elements needed for identification and visualization of the exemplar, it is appropriate to be used as a class-ancestor of experimental data. This will ensure compatibility with the object-oriented graphical extension.
For the category experiment $\boldsymbol{E}$ (7), a classheader TExperiment (Figure 2) as a successor of TMicroGraph is defined.

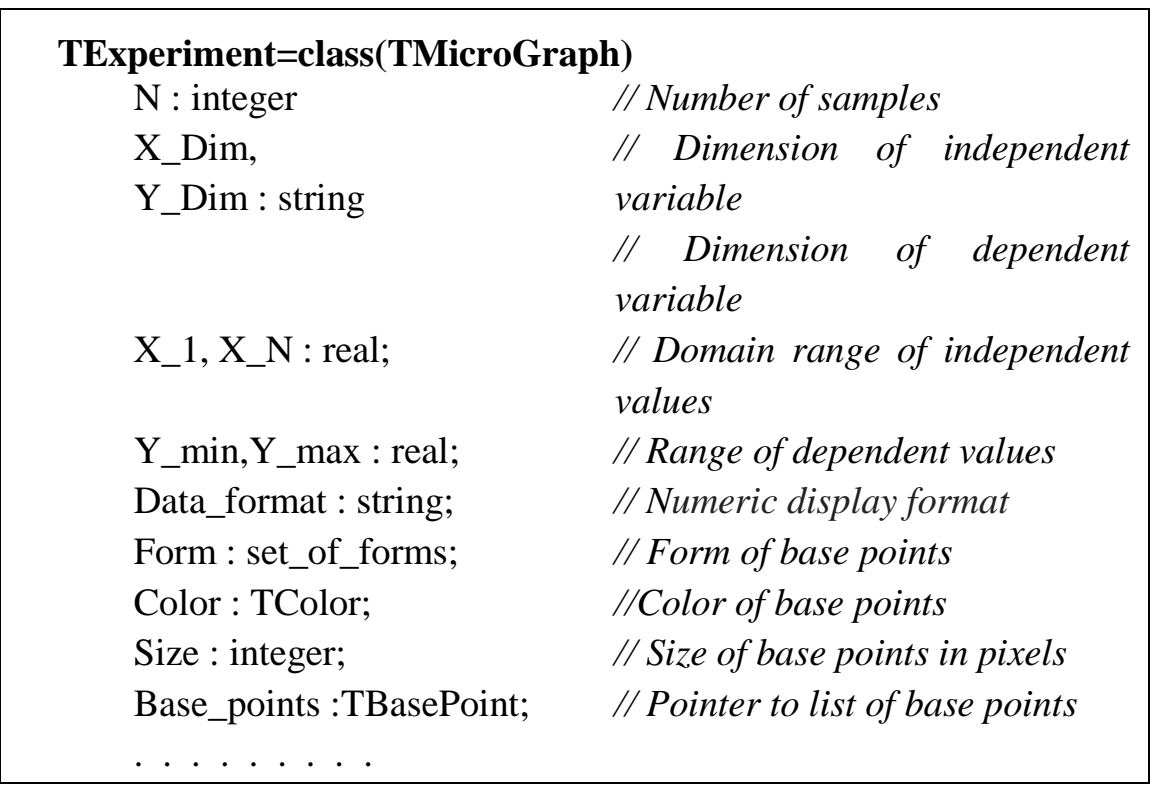

Figure 2. Data definition of Experiment

The pointer Base_points is the header (Figure

3) of a double-linked list with experimental base points: 


\begin{tabular}{|cl|}
\hline TBasePoint $=$ class(TObject) & \\
M : integer & // Serial number of base point \\
X, X_SD, & // Average value, standard \\
& deviation for X \\
Y, Y_SD : real; & // Average value, standard \\
& deviation for $Y$ \\
SKF $:$ real; & // approximation coefficient \\
Visible, & //visible/unvisible point \\
Active : boolean; & // active / inactive point \\
NextPoint, & // pointer to next base point \\
PrevPoint : TBasePoint; & // pointer to previous base point \\
. . . . . . . . & \\
\hline
\end{tabular}

Figure 3. Header-class TBasePoint

Besides the values of the ordered quaternion (8), TBasePoint contains approximation and visualization parameters for each base point. The boolean parameter "Active" determines whether the base point must be used in the calculation procedures (eg. approximation, differentiation, integration).

Each data class should have the realization part and interface part. The methods for data manipulation are described in the realization part. The interface part proposes a set of operations to access and modify the class properties.

$\checkmark$ Create and Destroy Methods. The constructor 'Create' allocates memory to instantiate the object and initializes its data.
The "Destroy" method destroys the class and releases the memory allocated to it.

$\checkmark$ GET operators. They are a necessary part to realize the class interface because of its encapsulation. They ensure access to the parameters of a concrete object in the program.

SET operators. These operators modify the properties of the classes.

$\checkmark$ File operations. They enable the writing in/reading from a file of experimental data. The possible data formats are ASCII, CSV, Paradox, SSP [1].

The structure of the formulated classes for presenting the experimental data is shown on Figure 4.

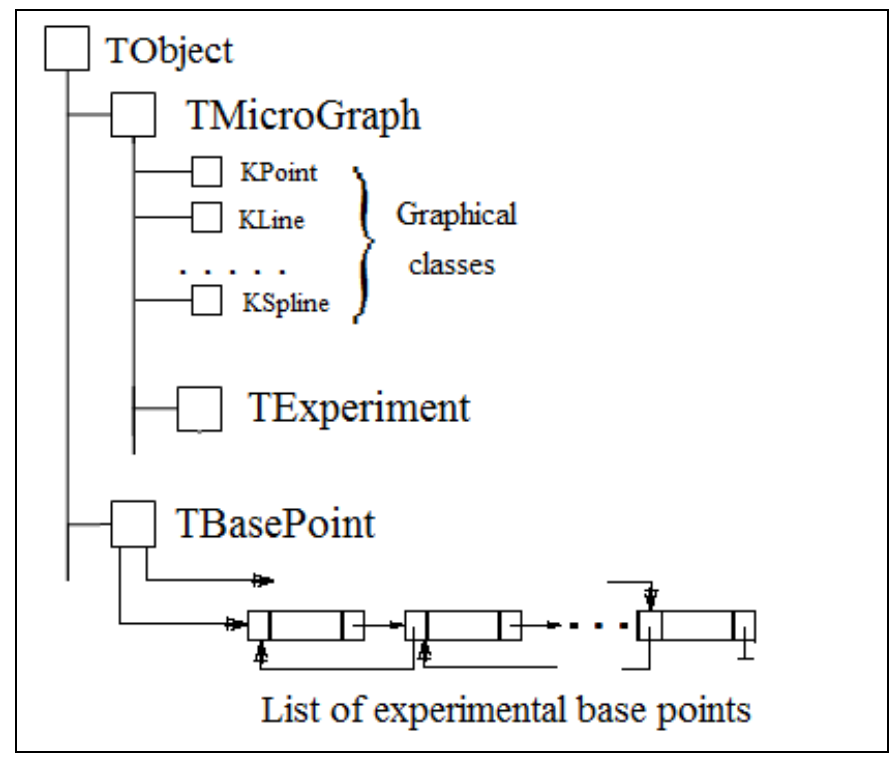

Figure 4. Class hierarchy of experimental data

\section{SOFTWARE REALIZATION}

The formulated requirements to the user interface and the supporting data structures are implemented in the program Korelia-Ident.
The program offers opportunities for data processing [4], identification [5], modeling, and studying of dynamic processes. With its 
user-friendly interface $[6,7]$, it is preferred by humanities scholars $[8,9,10]$.

In [11], how bread crust color changes during baking is modeled (with permission from $\mathrm{m}-\mathrm{r}$ Zl. Zlatev). Figure 5 shows a dialog with the data of the color components of the XYZmodel. In the same paper, the author uses Korelia-Ident to identify the color changes during baking.

\begin{tabular}{|c|c|c|c|c|c|c|c|}
\hline \multicolumn{2}{|l|}{ Ex } & & \multicolumn{4}{|c|}{ РЕДАКЦИЯ НА СПЛАЙН } & $-\square$ \\
\hline \multicolumn{2}{|c|}{ File name } & $\mathrm{E}: \mathrm{MyF}$ & rams\}SP & EZZlatin\}Br & d_Z.SSP & & \\
\hline \multirow{3}{*}{\multicolumn{2}{|c|}{$\begin{array}{c}\begin{array}{c}\text { Points } \\
\text { number }\end{array} \\
\begin{array}{c}\mathbf{\forall} \\
\text { decimal } \\
\text { digits }\end{array}\end{array}$}} & \multirow{2}{*}{$\begin{array}{r}\text { Xinit } \\
0.1300\end{array}$} & Step & \multirow{3}{*}{$2 \stackrel{\text { Size }}{2 \div}$} & \multicolumn{2}{|c|}{ Dimensions } & \multirow{3}{*}{$\begin{array}{l}\text { delete } \\
\ulcorner\text { unvisibl } \\
\ulcorner\text { inactive }\end{array}$} \\
\hline & & & & & \multirow{2}{*}{\multicolumn{2}{|c|}{$\begin{array}{cl}x & t, \text { Hours } \\
Y & \text { Z-component }\end{array}$}} & \\
\hline & & & & & & & \\
\hline No & $x$ & Y & $\times \mathrm{SD}$ & Y SD & visible & active & SKF \\
\hline 1 & 0.1300 & 0.8800 & 0 & 0.002 & 1 & 1 & 0.0000 \\
\hline 2 & 0.2500 & 0.6120 & 0 & 0.003 & 1 & 1 & 0.0054 \\
\hline 3 & 0.3800 & 0.5410 & 0 & 0.019 & 1 & 1 & -0.0011 \\
\hline 4 & 0.5100 & 0.4820 & 0 & 0.018 & 1 & 1 & 0.0004 \\
\hline 5 & 0.6300 & 0.4370 & 0 & 0.017 & 1 & 1 & 0.0010 \\
\hline 6 & 0.7600 & 0.4200 & 0 & 0.006 & 1 & 1 & -0.0015 \\
\hline 7 & 0.8800 & 0.3550 & 0 & 0.017 & 1 & 1 & 0.0000 \\
\hline 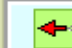 & $\Rightarrow$ 4 & Insert & & Save in File & OK & Cancel & \\
\hline
\end{tabular}

Figure 5. Data entry window

\section{CONCLUSIONS}

The article analyzes the nature and characteristics of the data obtained in scientific experiments. It was found that subject of statistics are not only the dependent, but also the independent variables. General laws and proposed dynamic structures for images and non-graphical data according to the requirements of Object-oriented programming are formalized.

A user-friendly interface for receiving and transforming data from DCE is developed, as well as interactive input of data into the computer system, accompanied by statistical processing and evaluation of data.

Basic structures are organized in classes according to the requirements of objectoriented programming. Abstract base class is TMicrograph. It is an ancestor of the TExperiment and TBasePoint classes. The last class forms dynamic double-linked list containing experimental base points.

The approach is implemented with the software Korelia-Ident. Korelia-Ident is intended to provide a highly interactive environment for researchers to identify, model and simulate the properties of dynamic experimental systems.

\section{ACKNOWLEDGMENTS}

This study was supported by Grant 04_ФТT/18.07.2014 “Interactive Program System for Identification and Evaluation of Experimental Data" from the Faculty of Technics and Technologies, Trakia University - Yambol, Bulgaria.

\section{REFERENCES}

1. Yankov, K. Interactive Generation of Data Transmission Protocol Between External Device and Computer. Proc. Int. Conference on Information Technologies (InfoTech-2007). sept.21-23, 2007, Varna, Bulgaria, pp 231-238.

2. Yankov, K. Structure and functions of the graphic programming languages. Proc. Eight Nat. Conf. "Modern Tendencies in the Development of Fundamental and Applied Sciences" june 5-6, 1997, Stara Zagora, Bulgaria, pp.94-102 (in Bulgarian).

3. Yankov, K. Object Oriented TwoDimensional Graphics in a Borland Delphi Environment. Proc. 13-th Int.Conf. SAER'99, sept.18-19, 1999, st.Konstantin resort, Varna, Bulgaria, pp. 169-173.

4. Yankov, K. Preprocessing of Experimental Data in Korelia Software. Trakia Journal of Sciences, Vol. 8, Suppl. 3, pp. 41-48, 2010

5. Yankov, K. System Identification of 
YANKOV K., et al.

Biological Processes. Proc. 20-th Int.Conf. "Systems for Automation of Engineering and Research (SAER-2006). St.St. Constantine and Elena resort, sept.23-24, 2006, Varna, Bulgaria, pp 144-149.

6. Yankov, K. Simple Expression Language for Model Identificaton. Proc. of the Int. Conference on Information Technologies (InfoTech-2008). Constantine and Elena resort, sept.19-21, 2008, Varna, Bulgaria, vol.2, pp.259-266.

7. Yankov, K. Recognition and Function Association of Experimental Data. Proc. of the Int. Conference on Information Technologies (InfoTech-2009). Constantine and Elena resort, sept.17-20, 2009, Varna, Bulgaria, pp.131-140.

8. Hadzhibozheva P.; A.Tolekova, Ts.Georgiev G.Ilieva, R.Kalfin. Angiotensin II Receptors Type 2 and Gastrointestinal Tract Contractile Activity.
Comptes rendus de l'Acadmie Bulgare des Sciences. Tome 67, No 8, 2014. pp. 10911100.

9. Hadzhibozheva P.V., T.K. Georgiev , R.E.Kalfin , A.N. Tolekova. Angiotensin II and Vasopressin Effects on Motor Activity of Rat Isolated Tissue Strips from Urinary Bladder and Rectum. Bulg. Chem. Commun., 2012 , 44 (3), $252-257$.

10.Hadzhibozheva P. V., A. N. Tolekova, T. K. Georgiev. Angiotensin II receptors - role for the contraction of large intestine. Bulgarian Journal of Veterinary medicine, 2013, 16, Supp. 1, 10-18.

11.Zlatev Z. Modeling of Color Changes in Bread Crust During Baking. Proc. Int. Conf. on Technics, Technologies and Education "ICTTE 2014", oct. 30-31 2014, Yambol, Bulgaria. pp.680-685. 